\title{
Development of a novel tissue-engineered nitinol frame artificial trachea with native-like physical characteristics
}

Yasuto Sakaguchi, MD, ${ }^{\mathrm{a}, \mathrm{b}}$ Toshihiko Sato, MD, PhD, ${ }^{\mathrm{b}, \mathrm{c}}$ Yusuke Muranishi, MD, ${ }^{\mathrm{a}, \mathrm{b}}$ Yojiro Yutaka, MD, ${ }^{\mathrm{a}, \mathrm{b}}$

Teruya Komatsu, MD, ${ }^{\mathrm{a}, \mathrm{b}}$ Koichi Omori, $\mathrm{MD}, \mathrm{PhD},{ }^{\mathrm{d}}$ Tatsuo Nakamura, $\mathrm{MD}, \mathrm{PhD},{ }^{\mathrm{a}}$ and

Hiroshi Date, $\mathrm{MD}, \mathrm{PhD}^{\mathrm{b}}$

\section{ABSTRACT}

Background: Tracheal reconstruction is complicated by the short length to which a trachea can be resected. We previously developed a biocompatible polypropylene frame artificial trachea, but it lacked the strength and flexibility of the native trachea. In contrast, nitinol may provide these physical characteristics. We developed a novel nitinol frame artificial trachea and examined its biocompatibility and safety in canine models.

Methods: We constructed several nitinol frame prototypes and selected the frame that most closely reproduced the strength of the native canine trachea. This frame was used to create a collagen-coated artificial trachea that was implanted into 5 adult beagle dogs. The artificial trachea was first implanted into the pedicled omentum and placed in the abdomen. Three weeks later, the omentum-wrapped artificial trachea was moved into the thoracic cavity. The thoracic trachea was then partially resected and reconstructed using the artificial trachea. Follow-up bronchoscopic evaluation was performed, and the artificial trachea was histologically examined after the dogs were sacrificed.

Results: Stenosis at the anastomosis sites was not observed in any dog. Survival for 18 months or longer was confirmed in all dogs but 1 , which died after 9 months due to reasons unrelated to the artificial trachea. Histological examination confirmed respiratory epithelial regeneration on the artificial trachea's luminal surface. Severe foreign body reaction was not detected around the nitinol frame.

Conclusions: The novel nitinol artificial trachea reproduced the physical characteristics of the native trachea. We have confirmed cell engraftment, good biocompatibility, and survival of 18 months or longer for this artificial trachea in canine models. (J Thorac Cardiovasc Surg 2018;156:1264-72)

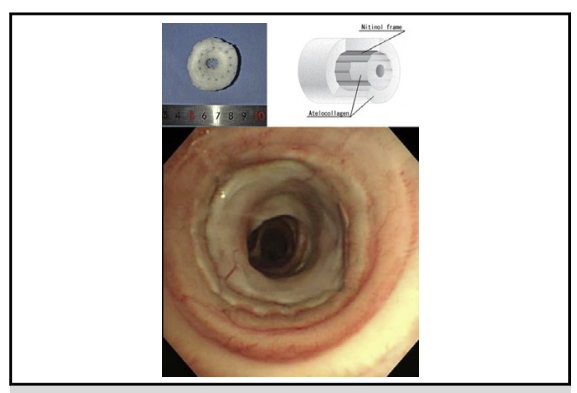

A novel collagen-coated nitinol frame artificial trachea implanted in a canine model.

\section{Central Message}

This novel collagen-coated nitinol frame artificial trachea reproduced the physical properties of the native trachea. Good biocompatibility was confirmed in canine models.

\section{Perspective}

A collagen-coated nitinol frame was used to produce an artificial trachea with similar physical characteristics as the native trachea. This novel artificial trachea was implanted in dogs, where it demonstrated cell engraftment, good biocompatibility, and survival of 18 months or longer. These findings represent the first step toward the clinical application of this artificial trachea in human patients.

See Editorial Commentary page 1273.

See Editorial page 1263
Tracheal reconstruction is sometimes indicated in the surgical management of various benign and malignant tumors. However, there is a limit to the length to which a trachea

From the a Department of Bioartificial Organs, Institute for Frontier Life and Medical Sciences, Kyoto University, Kyoto, Japan; and ${ }^{\mathrm{b}}$ Department of Thoracic Surgery, ${ }^{\mathrm{c}}$ Institute for Advancement of Clinical and Translational Science, and ${ }^{\mathrm{d}}$ Department of Otolaryngology Head and Neck Surgery, Kyoto University Hospital, Kyoto, Japan.

Received for publication Jan 29, 2018; revisions received March 15, 2018; accepted for publication April 15, 2018; available ahead of print May 18, 2018.

Address for reprints: Toshihiko Sato, MD, PhD, Institute for Advancement of Clinical and Translational Science, Kyoto University Hospital, 54 Shogoin-Kawahara-cho, Sakyo-ku, Kyoto 606-8507, Japan (E-mail: tsato@ kuhp.kyoto-u.ac.jp). $0022-5223 / \$ 36.00$

Copyright (c) 2018 by The American Association for Thoracic Surgery https://doi.org/10.1016/j.jtcvs.2018.04.073 can be resected and reconstructed. Previous studies have reported that a $6-\mathrm{cm}$ resection is the maximum length that allows subsequent anastomosis of the native trachea with current methods. ${ }^{1,2}$ To overcome this limitation, the use of artificial tracheae has been extensively examined. Through in situ tissue engineering, we previously developed an artificial trachea in which a polypropylene mesh was molded

- Scanning this $\mathrm{QR}$ code will take you to the supplemental videos for the article.

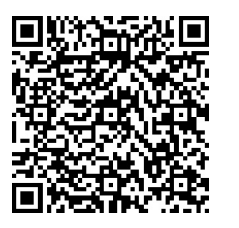




\section{Abbreviation and Acronym \\ $\mathrm{CT}=$ computed tomography}

into a cylinder and coated with an atelocollagen layer. ${ }^{3-9}$ Our experiments demonstrated that this collagen coating promotes regeneration of the autologous respiratory epithelium, which prevents exposure of the artificial trachea's luminal surface to external conditions. ${ }^{3-9}$ This polypropylene frame artificial trachea demonstrated longterm safety and biocompatibility, and was successfully applied in a clinical setting ${ }^{5}$; however, it was unable to reproduce the strength, flexibility, and shape recovery properties of the native trachea for stabilizing the lumen against external forces.

To address these shortcomings, we focused on the use of nitinol as an alternative material for constructing artificial tracheae. Nitinol is a shape memory alloy that provides restoring forces against deformations resulting from external stress. Because of this characteristic, nitinol is used not only in industrial settings, but also in medical applications, such as the development of airway and vascular stents. A major advantage of using nitinol in stent production is that it enables the specification of material strength through changes in wire diameter and knitting patterns. We considered that this attribute may have similar applications in the development of artificial tracheae with physical properties that approximate those of the native trachea. Here we describe the development of a novel artificial trachea composed of a nitinol frame that reproduces the physical characteristics of the native trachea. The artificial trachea was coated with collagen using previously described techniques, ${ }^{3-9}$ and we examined the biocompatibility and issues of this artificial trachea through implantation experiments in canine models.

\section{METHODS}

\section{Development of the Artificial Trachea} Quantifying the mechanical properties of the native trachea. The native tracheal strength of adult beagle dogs was first measured to quantify the trachea's mechanical properties. Thoracic tracheae were collected from 5 dogs that had been sacrificed for another study. The mean length, width of the membranous portion, and distance from the ventral to the dorsal sides of the harvested tracheae were $80 \mathrm{~mm}, 22 \mathrm{~mm}$, and $20 \mathrm{~mm}$, respectively.

To measure the mechanical strength of the native trachea, each harvested trachea was placed between a force gauge (RZ-5; Aikoh Engineering, Osaka, Japan) and a cradle. The component of the force gauge that contacts the trachea measured $1.5 \times 5.0 \mathrm{~cm}^{2}$, and the long axis of this component was placed perpendicular to the long axis of the trachea at the center of the cradle. The load applied to the force gauge was measured by raising the cradle, which moved from the posterior side of the trachea toward the anterior side (Figure 1, $A$ ). The load was measured 5 times for each sample, and these measurements were used to determine the strength required in the artificial trachea. The fitted curves of the mechanical strength measurements were generated using JMP 13 (SAS Institute, Cary, NC).
Constructing the nitinol frame. To construct the artificial tracheal frame, we used nitinol wire $(0.18 \mathrm{~mm}$ diameter $)$ composed of $55.9 \%$ nickel and $44.1 \%$ titanium (KIOKALLOY-R; Daido Steel, Nagoya, Japan). The shape recovery temperature of this alloy ranges between $20^{\circ} \mathrm{C}$ and $100^{\circ} \mathrm{C}$, making it appropriate for in vivo use. The nitinol wire was molded into a continuous cylindrical shape using spool knitting. The cylindrical-shaped frame was heat-treated at $500^{\circ} \mathrm{C}$ for 15 minutes and then cooled with water (Marui Textile Machinery, Osaka, Japan). Two types of nitinol frames were initially fabricated: one with equal-width stitches (type A: $1.25-\mathrm{mm}$ stitches) and another with unequal-width stitches (type B: 1.25-mm and 4.5-mm stitches) (Figure 1, B). The mechanical strength of both frames was quantified using the same method as for the native tracheae (Figure 2), and the type B frame was found to be stronger. However, the type B frame was still weaker than the native trachea. To more closely reproduce the native tracheal strength, another frame (type C; Figure $1, B$ ) was developed with unequal stiches similar to type B but with smaller stitches in the major axis direction $(0.75-\mathrm{mm})$. Because the type $\mathrm{C}$ frame had sufficient mechanical strength that was closest to that of the native trachea (Figure 2), this frame was chosen for use in the subsequent tracheal reconstruction experiments. The type $\mathrm{C}$ nitinol frame was shown to stabilize the lumen against elongation, twisting, and bending (Video 1).

Constructing the artificial trachea. We used a nitinol frame with a length of $30 \mathrm{~mm}$ and a diameter of $22 \mathrm{~mm}$ to construct each artificial trachea. The outer and inner surfaces of the nitinol frame were coated with $10 \mathrm{~mm}$ and $8 \mathrm{~mm}$ of $3 \%$ atelocollagen, respectively (Figure 3 ). ${ }^{10,11}$ The collagen was frozen for 24 hours at $-80^{\circ} \mathrm{C}$ and then freeze-dried under vacuum for 48 hours before undergoing thermal cross-linking for 12 hours at $140^{\circ} \mathrm{C}$. The collagen was then sterilized before use.

\section{Implanting the Artificial Trachea and Reconstructing the Trachea}

The implantation surgery was performed in 5 adult beagle dogs in 2 stages (Figure 4). In the first surgery, laparotomy was performed under general anesthesia. A ventral midline incision of $5 \mathrm{~cm}$ was made, and the pedicled omentum was pulled to the outside of the abdominal cavity. The artificial trachea was wrapped in the omentum, which was then replaced into the abdominal cavity (Figure 4, $A$ and $D$ ). This first surgery was aimed at preventing air leakage and infection of the artificial trachea through omentum wrapping.

The second surgery was performed 3 weeks after the first surgery using the same laparotomic approach. First, we confirmed that the artificial trachea was wrapped in the omentum. A hole was made in the right diaphragm, and the omentum-wrapped artificial trachea was guided from the abdominal cavity into the right thoracic cavity. Through the right thoracotomy, $20 \mathrm{~mm}$ of the thoracic trachea was resected (Figure 4, $B$ and $E$ ) from the position where 3 to 4 cartilage rings remained from the tracheal bifurcation. Ventilation was provided via endotracheal tubes, and telescopic anastomosis was performed such that the native trachea was inserted $5 \mathrm{~mm}$ inside the artificial trachea. The segment bridged by the artificial trachea was $20 \mathrm{~mm}$ long (Figure 4, $C$ and $F$ ). After checking for air leaks, the mediastinal pleura was sutured. The right lung was reexpanded through pressurization with a ventilator at chest closure, and the surgery was completed.

\section{Evaluation}

Follow-up observations were performed at 1, 3, 6, 9, 12, 18, and 24 months after the second surgery via bronchoscopy and computed tomography (CT) scans. The dogs were sacrificed at 18 and 24 months after the second surgery, and the artificial tracheae were resected with the surrounding tissues in en bloc fashion. After formalin fixation, the tissue blocks were cut along the longitudinal direction of the trachea such that the cut face of the nitinol frame was exposed. The excised tissues were embedded in paraffin, and the nitinol frame was removed from one side. Sections were prepared from the tissue surface on the opposite side from 


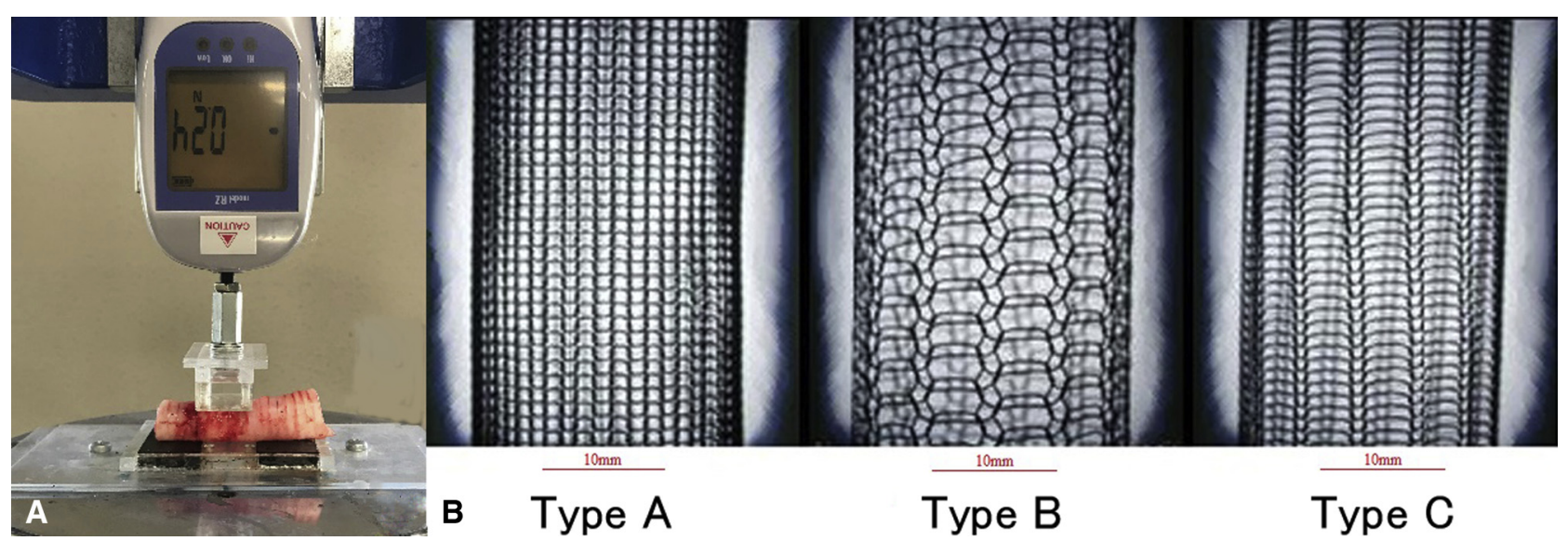

FIGURE 1. Strength-measuring device and nitinol frame prototypes for the artificial trachea. A, To measure tracheal strength, each trachea was placed between a force gauge and a cradle. The load pressure applied to the force gauge was measured by raising the cradle. B, All nitinol frames were woven from a single wire. The type A frame was made with equal-width stitches (1.25-mm stitches), and the type B frame was made with unequal-width stitches (1.25-mm and 4.5-mm stitches). The type $\mathrm{C}$ frame was also made with unequal-width stitches (similar to type $\mathrm{B}$ ), but with smaller stitches in the major axis direction $(0.75-\mathrm{mm})$.

which the nitinol frame was removed and examined microscopically after staining with hematoxylin and eosin.

We compared our present experimental results of implantation of the nitinol frame artificial trachea with our previously reported results with the polypropylene frame artificial trachea. ${ }^{3,8}$ In those previous studies, we evaluated the duration of postimplantation survival, bronchoscopic findings of the anastomosis site, and regeneration of the ciliated respiratory epithelium on the artificial tracheal lumen. ${ }^{3,8}$ In the present analysis, we evaluated the same 3 outcomes for the nitinol frame artificial trachea.

\section{Animal Experiments and Care}

Adult beagle dogs (body weight, 8.7-11.5 kg) were used in this series of experiments. All surgical procedures were performed by a board-certified surgeon. For anesthesia during surgery, each dog was pretreated with $0.03 \mathrm{mg} / \mathrm{kg}$ of atropine sulfate and sedated using an intramuscular injection of $15 \mathrm{mg} / \mathrm{kg}$ ketamine hydrochloride and $7 \mathrm{mg} / \mathrm{kg}$ xylazine. Intubation was performed, and general anesthesia was maintained with sevoflurane and oxygen. The dogs were later euthanized using deep anesthesia and an overdose of sodium pentobarbital.

All animal experiments were performed with the approval of the Committee for Animal Research of Kyoto University. All animals were treated humanely in compliance with the eighth edition of the Guide for the Care and Use of Laboratory Animals published by the National Institutes of Health.

\section{RESULTS}

\section{Postoperative Survival}

The postoperative survival of the $5 \mathrm{dogs}$ is presented in Table 1. Survival for 18 months or longer after the second surgery was confirmed in all dogs but 1 ; the longest survival duration was 24 months. One dog died suddenly after 9 months with no specific symptoms before the event. Necropsy examination of this dog revealed no evidence of airway obstruction or anastomotic leakage, or any bleeding or infection resulting from contact between the nitinol frame and the surrounding tissues and blood vessels. There were no indications that implantation of the artificial trachea was directly associated with this dog's death.

\section{Bronchoscopic and CT Findings}

Bronchoscopic examination did not detect airway stenosis in any of the surviving cases at 18 months (Table 1). Some mucosal irregularities were observed at the anastomosis sites at 1 month after the second surgery, but these eventually receded (Fig. 5, A-D). In the month after the second surgery, all dogs exhibited $90 \%$ epithelialization (Table 1), and this epithelialization remained stable at 24 months (Video 2). Exposure of the nitinol frame to the endotracheal cavity was not observed. Angiogenesis originating from the native trachea was not detected at 1 month after the second surgery, but was observed in the lumen of the artificial trachea after 3 months in 3 dogs (Figure 5, E). Mild granulation was observed at the peripheral anastomosis site in $1 \mathrm{dog}$ (Figure 5, F), and scar formation was observed after ulceration in a portion of the artificial endotracheal lumen in another dog (Figure 5, G). Neither of these conditions necessitated treatment for airway stenosis, such as stent insertion. Follow-up CT examination did not show any signs of pneumonitis, inflammation, or erosion in the surrounding tissue (eg, esophagus) due to the artificial trachea. In addition, the nitinol frame was not exposed in any of the cases. Airways were shown to be sufficiently patent in a 3-dimensional reconstruction of CT images (Figure 5, $H$ ).

\section{Histological Findings}

Macroscopic examination revealed no tracheal stenosis at the anastomosis sites, and the luminal surface of the artificial trachea was smooth (Figure 6, A). Microscopic examination of the anastomosis sites showed that the tracheal epithelium continued smoothly from the native trachea to the artificial trachea, but the muscular layer had not 


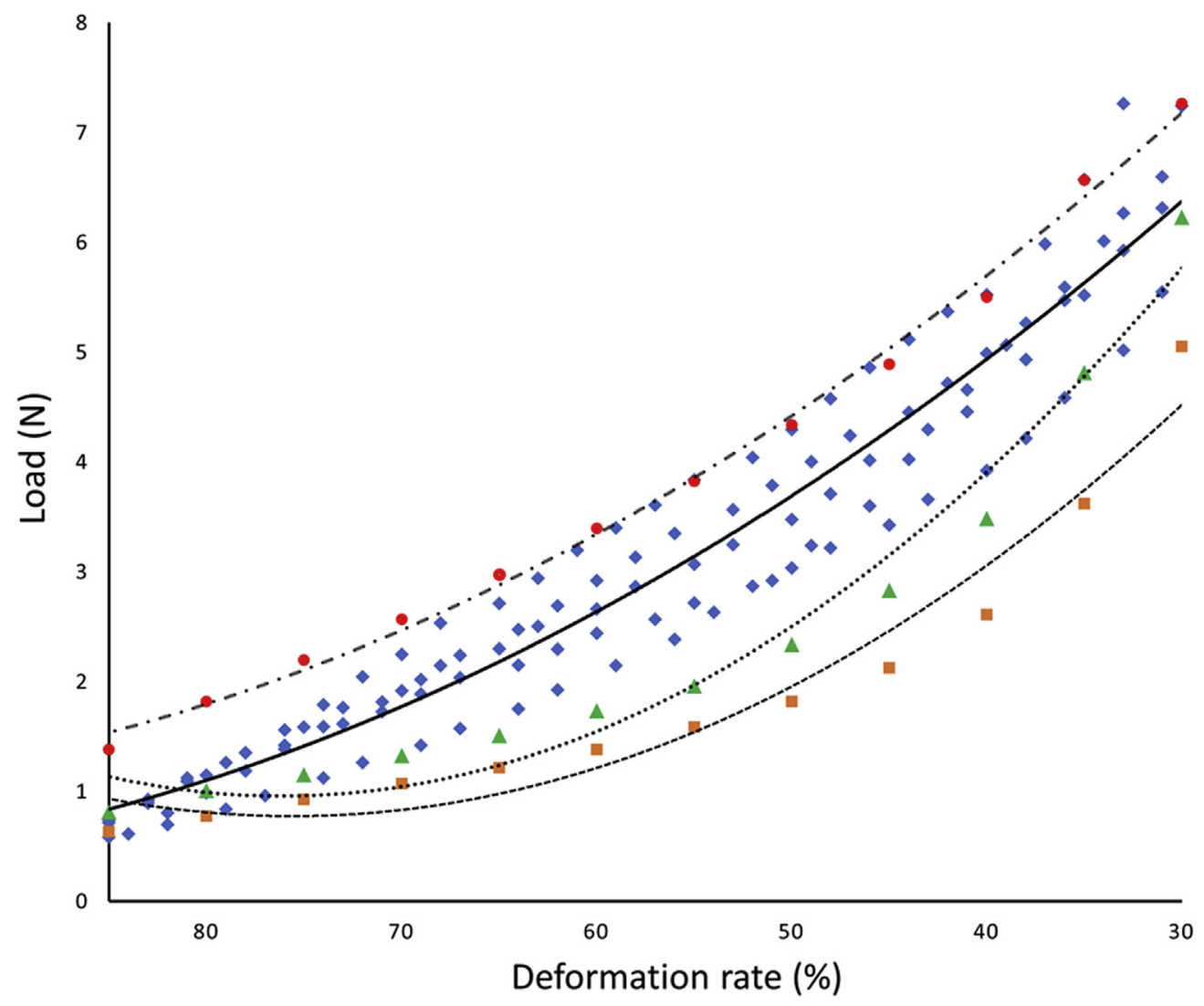

$\rightarrow$ Native trachea

$\triangle$ Type B frame

-Fitted curve of native trachea

.... Fitted curve of Type B frame
- Type A frame

- Type C frame

---Fitted curve of Type A frame

- Fitted curve of Type $C$ frame

FIGURE 2. Strength measurement results for the native tracheae and nitinol frames. In this strength comparison graph, the vertical axis represents the load measured with the force gauge. The horizontal axis indicates the change rate of the height of the lumen in the vertical direction. Assuming that the height without load is $100 \%$, the state in which the height is halved is $50 \%$, and the state in which the height is completely closed is $0 \%$. All measurements were performed 5 times, and the mean value was calculated. The type B frame demonstrated higher strength than the type A frame but was still considerably weaker than the native trachea. The strength of the type $\mathrm{C}$ frame was comparable to that of the native trachea, and the fitted curves of both the native trachea and the type $\mathrm{C}$ frame had similar slopes (native trachea: $y=0.0010 x^{2}-0.2107 x+11.8340, R^{2}=0.9301$; type A frame: $y=0.0018 x^{2}-0.2720 x+11.0622$, $R^{2}=0.9525$; type B frame: $y=0.0023 x^{2}-0.3437 x+14.0452, R^{2}=0.9722$; type C frame: $y=0.0010 x^{2}-0.2189 x+12.8379, R^{2}=0.9961$ ).

regenerated in the latter (Figure 6, $B$ ). Histological examination confirmed the regeneration of the ciliated respiratory epithelium on the artificial trachea. However, the regenerated epithelium presented as a monolayer, which differed from the stratified layer of the native tracheal epithelium (Figure 6, $C$ and $D$ ). In contrast to the native tracheal epithelium, tracheal glands were not observed in the monolayer epithelium (Figure 6, $C$ and $D$ ). Slight inflammation and scarring were observed around the nitinol frame (Figure 6, E).

\section{DISCUSSION}

The novel nitinol frame artificial trachea that we have developed closely reproduces the strength, flexibility, and shape recovery properties of native trachea. Our experiments indicate that this artificial trachea has good biocompatibility with long-term survival in canine models. These findings support the use of the nitinol frame artificial trachea for tracheal reconstruction and represent the first step toward its clinical application.

Tracheal reconstruction is an important procedure in the fields of thoracic and otolaryngologic surgery. This surgery requires high levels of operator skill and experience owing to limitations associated with tracheal anastomosis. In a simple anastomosis, a tracheal resection of $2.0 \mathrm{~cm}$ is generally considered acceptable. ${ }^{2}$ Although it is possible to increase this length to $6.0 \mathrm{~cm}$ in staged operations, this involves complicated procedures with their attendant 


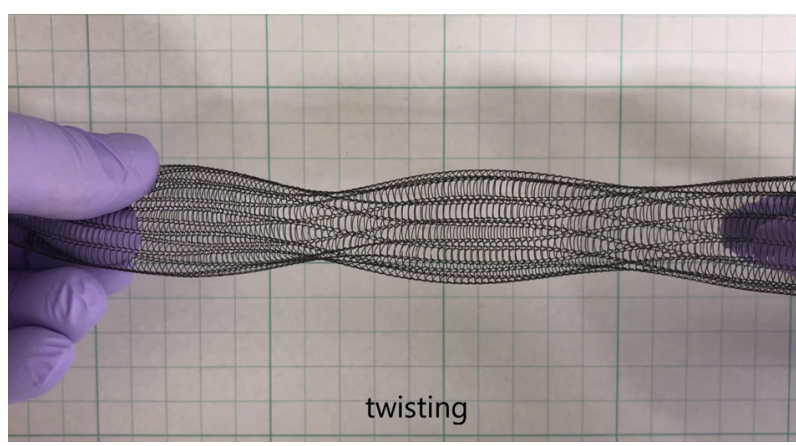

VIDEO 1. The nitinol frame stabilizes the lumen against elongation, twisting, and bending forces. The frame reverts to its original shape when these forces are removed. Stabilization of the lumen against external forces is an important physical characteristic of the native trachea. Video available at: https://www.jtcvs.org/article/S0022-5223(18)31211-X/fulltext.

risks. ${ }^{1,2}$ For these reasons, researchers have examined the feasibility of artificial tracheae created from various materials, such as homogeneous tissues, ${ }^{12-16}$ heterogeneous tissues, ${ }^{17}$ prosthetic materials, ${ }^{18,19}$ and combined materials. ${ }^{20,21}$ Various degrees of success have been reported, but the use of these materials generally involves complicated or time-consuming processes to prepare the artificial tracheae. The widespread clinical use of artificial tracheae requires long-term safety, efficacy, and a stable supply of materials, similar to the use of polyurethane and polytetrafluoroethylene in the production of artificial blood vessels. ${ }^{22,23}$ We had previously developed a biocompatible polypropylene frame artificial trachea, ${ }^{3-9}$ but found that it lacked the strength and elasticity of the native trachea.

An artificial trachea with similar physical properties as native trachea has several advantages. Because the artificial trachea can follow the movement of each tracheal stump in the cranial and caudal sides, the force applied to the anastomosis site can be reduced. During coughing or expectoration, the shape of the native trachea lumen changes due to increases in intrathoracic pressure, and these changes can be reproduced by the artificial trachea. By changing the shape of the artificial trachea, the influence on the surrounding tissue can be reduced. This may be useful in preventing fistula formation between the artificial trachea and the surrounding tissue.

Unlike polypropylene, nitinol has these physical characteristics. Nitinol is a shape memory alloy of nickel and titanium that maintains its configuration at room temperature after being formed into the desired shape and baked. ${ }^{24-27}$ This alloy also has been reported to have good biocompatibility, and may be suitable for in vivo implantation. ${ }^{26,27}$ In the medical field, nitinol is currently used in the production of coronary, airway, esophageal, and biliary stents. ${ }^{24,26,27}$ These nitinol stents are used in areas where long-term stability, flexibility, and shape maintainability are required, which are also important attributes for artificial tracheae.

In this study, modifications to the knitting pattern of the cylindrical nitinol frame provided it with comparable strength to native trachea, and we confirmed that the slopes of the fitted curves of strength measurements were very similar in the type $\mathrm{C}$ frame and native trachea. It is crucial that the artificial trachea have sufficient strength to maintain adequate patency in response to sudden increases in intrathoracic pressure during coughing or expectoration. The intrathoracic pressure during coughing can exceed $100 \mathrm{mmHg},{ }^{28}$ during which the patency rate is approximately $50 \%$ in the anteroposterior diameter of the trachea. ${ }^{29}$ A pressure of $100 \mathrm{mmHg}$ corresponds to a force of $1.36 \mathrm{~N} / \mathrm{cm}^{2}$. Assuming an area of contact of the native trachea to the sensor component during strength measurement of $3.0 \mathrm{~cm}^{2}$, the resulting force would be $4 \mathrm{~N}$. As shown in Figure 2, the patency rate of the native trachea at $4.0 \mathrm{~N}$ was close to $50 \%$. The strength measurements indicated that the type A and B frames would not maintain a $50 \%$ patency rate during coughing, which precluded their use in artificial tracheae. In contrast, the type $\mathrm{C}$ frame demonstrated sufficient strength to maintain a patency rate similar to that of the native trachea. The nitinol frame was also able to revert to its original shape after exposure to elongation, twisting, and bending forces. These properties allowed the
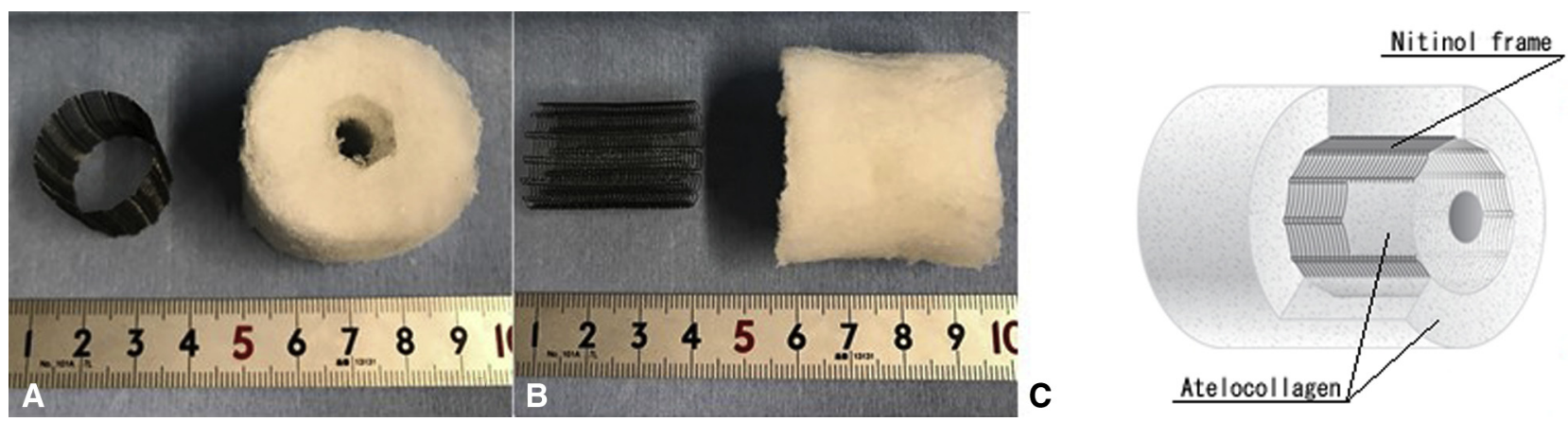

FIGURE 3. Characteristics of the nitinol frame for the artificial trachea. A, The nitinol frame was $22 \mathrm{~mm}$ in diameter. B, The nitinol frame was $30 \mathrm{~mm}$ long. $\mathrm{C}$, The inner and outer layers of the frame were coated with $3 \%$ atelocollagen. 

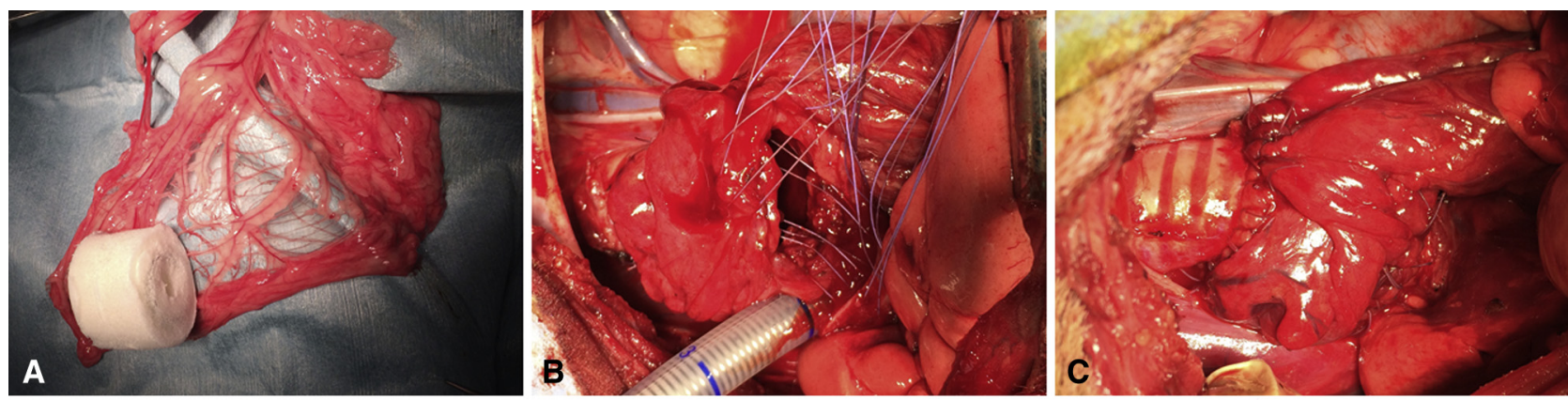

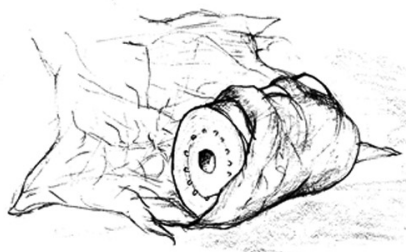

D

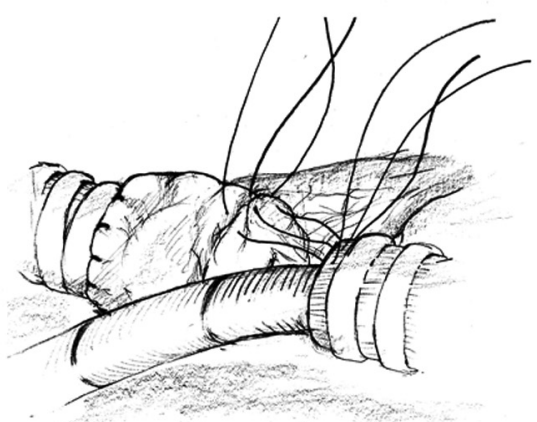

E

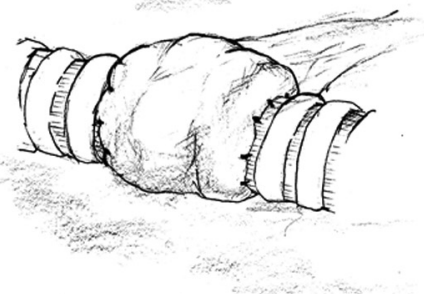

F

FIGURE 4. Implantation of the artificial trachea into the omentum and subsequent anastomosis with the thoracic trachea. A, B, and $\mathrm{C}$ are photos from the first and second surgeries; D, E, and F are schemata of the first and second surgeries. A, The artificial trachea before wrapping in the pedicled omentum. B, Anastomosis of the artificial trachea through surgical field intubation. C, State of the wrapped artificial trachea after anastomosis. D, In the first surgery, laparotomy was performed with a ventral midline incision of $5 \mathrm{~cm}$. The artificial trachea was wrapped in the pedicled omentum and inserted into the abdomen. E, In the second surgery, the omentum-wrapped artificial trachea was moved from the abdomen to the right thoracic cavity via the substernal route. F, Telescopic anastomosis was performed such that the native trachea overlapped 5 mm inside the artificial trachea. The final portion bridged by the artificial trachea measured $20 \mathrm{~mm}$.

novel artificial trachea to reproduce the physical characteristics of the native trachea, which is critical for longsegment tracheal reconstruction. In our experiment, 4 of the 5 dogs implanted with the artificial trachea survived for 18 months or longer after tracheal reconstruction.

Bronchoscopic examination did not reveal any signs of anastomotic stenosis in the nitinol frame artificial tracheae, similar to our previous findings with the polypropylene frame artificial tracheae. ${ }^{3,8}$ Angiogenesis on the artificial trachea's luminal surface was observed in 3 of the dogs in 3 months, as was also reported in our previous study of the polypropylene frame artificial trachea. ${ }^{8}$ Epithelialization had occurred at a rate of $20 \mathrm{~mm} / \mathrm{month}$ after the second surgery, but angiogenesis was not confirmed during the first month. Therefore, our findings suggest that angiogenesis required approximately 2 months before originating from the native trachea. Angiogenesis is an important indicator of engraftment in the artificial trachea. Although the regeneration of the ciliated epithelium on the luminal surface was confirmed, it appeared as a monolayer instead of a stratified ciliated columnar epithelium. In addition, no tracheal glands were detected. The respiratory epithelial regeneration in the nitinol frame artificial trachea was similar to that observed in the polypropylene frame artificial trachea. $^{3,8}$ Microscopic examination revealed only slight inflammation and scarring around the artificial trachea,

TABLE 1. Postoperative survival and bronchoscopic findings

\begin{tabular}{|c|c|c|c|c|c|c|c|c|}
\hline \multirow[b]{2}{*}{ Dog } & \multirow[b]{2}{*}{ Stenosis } & \multirow[b]{2}{*}{ Anastomosis site } & \multirow{2}{*}{$\begin{array}{l}\text { Exposure of the } \\
\text { nitinol frame }\end{array}$} & \multirow[b]{2}{*}{ Luminal surface } & \multicolumn{2}{|c|}{ Epithelialization, \% } & \multirow[b]{2}{*}{ Angiogenesis } & \multirow[b]{2}{*}{ Remarks } \\
\hline & & & & & $1 \mathrm{mo}$ & $3 \mathrm{mo}$ & & \\
\hline 1 & - & Mild granulation & - & Smooth & 90 & 100 & $3 \mathrm{mo}$ & Sacrificed at 24 mo. \\
\hline 2 & - & Smooth & - & Smooth & 100 & 100 & $3 \mathrm{mo}$ & Sudden death at 9 mo. \\
\hline 3 & - & Smooth & - & Smooth & 90 & 100 & - & Sacrificed at $18 \mathrm{mo}$. \\
\hline 4 & - & Smooth & - & Smooth & 90 & 100 & - & Sacrificed at 24 mo. \\
\hline 5 & - & Smooth & - & Partial ulcer scar & 100 & 100 & $3 \mathrm{mo}$ & Sacrificed at $18 \mathrm{mo}$. \\
\hline
\end{tabular}




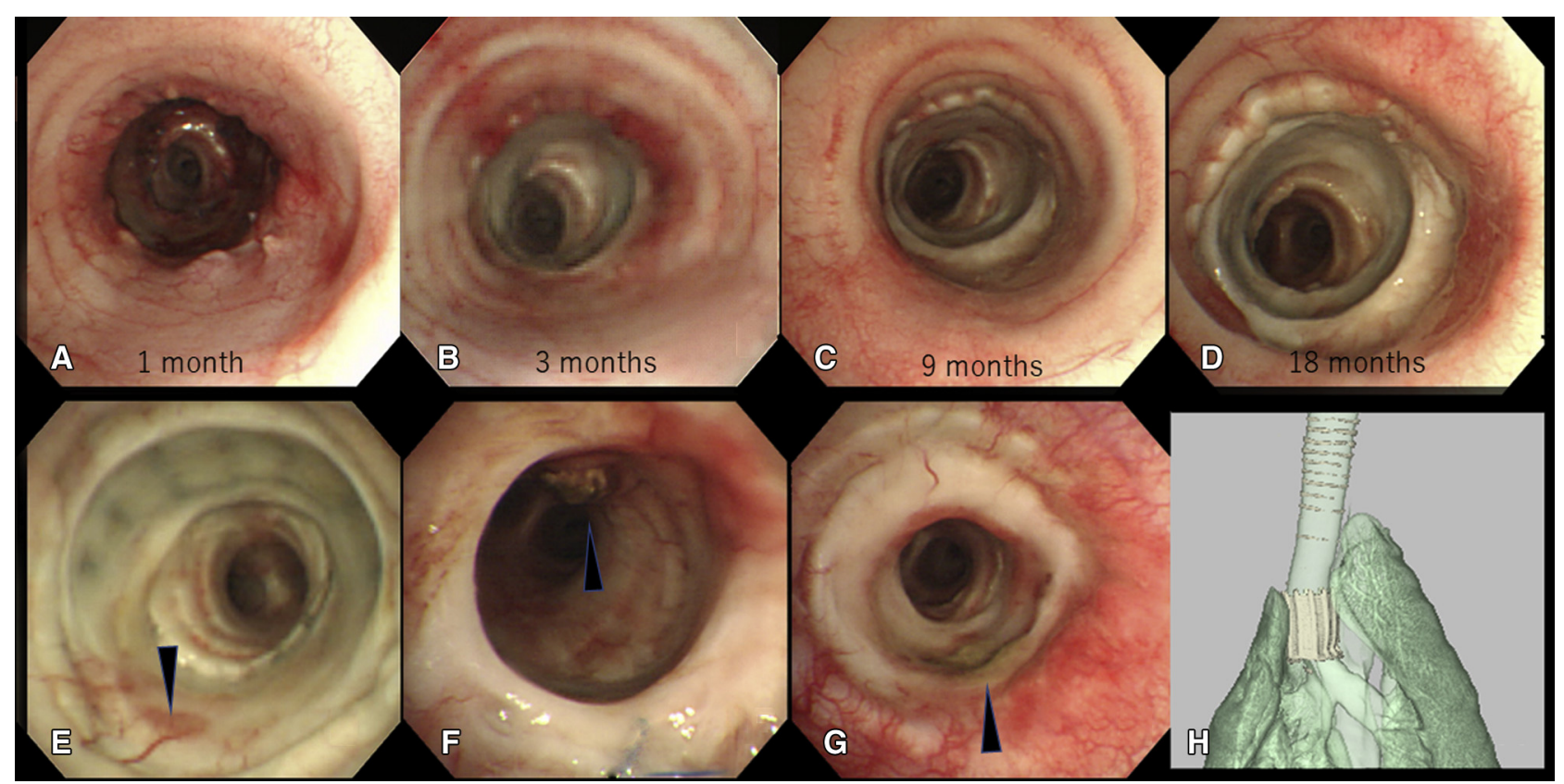

FIGURE 5. Bronchoscopic findings and 3-dimensional reconstructed computed tomography scans following artificial trachea implantation. A, Bronchoscopic examination showing mucosal irregularities at the anastomosis site 1 month after the second surgery. B, C, and D, The previously observed irregularities at the anastomosis site receded between 3 and 18 months. E, New blood vessels (arrow) had formed in the artificial trachea from the native trachea. F, Mild granulation (arrow) was observed at the peripheral anastomosis site. G, Scar formation (arrow) was observed after ulceration in a portion of the artificial endotracheal lumen. $\mathrm{H}$, Three-dimensional reconstructed computed tomography scan showing the artificial trachea fixed on the tracheal bifurcation. The nitinol frame was not exposed to external conditions in all cases.

suggesting that there was no severe rejection of the nitinol frame. The long survival durations, bronchoscopic findings, and microscopic findings indicate that the nitinol

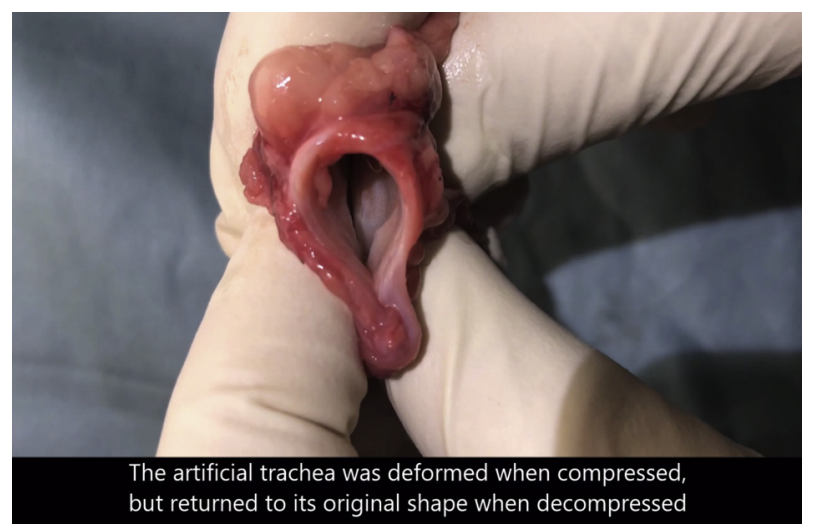

VIDEO 2. Follow-up bronchoscopy was performed at 24 months after the second surgery. Epithelialization was observed in the lumen of the artificial trachea. After resection of the engrafted artificial trachea, manual pressure was applied to the engrafted artificial trachea. The artificial trachea was deformed when compressed but returned to its original shape when decompressed. If pressure were applied from the surroundings during a cough, it is conceivable that the artificial trachea would deform synchronously with the native trachea. Video available at: https://www.jtcvs.org/article/S00225223(18)31211-X/fulltext. frame artificial trachea had a high level of biocompatibility comparable to that of our polypropylene frame artificial trachea. This biocompatibility also suggests that the nitinol frame artificial trachea has potential clinical applications for in situ tissue engineering in humans.

To prevent air leakage and infection, the nitinol frame artificial trachea was embedded in the pedicled omentum at 3 weeks before the second surgery, and the omentumwrapped artificial trachea was guided to the right thoracic cavity during the second surgery. This step is also important for encouraging the engraftment of the artificial trachea, because the omentum wrapping ensures blood flow and promotes revascularization, which can affect the success of implantation. ${ }^{2}$ Wrapping the artificial trachea with a pedicled omental flap is a common procedure for ensuring blood flow and revascularization, and we have previously used this technique for intrathoracic tracheal reconstruction. ${ }^{6}$ Whereas angiogenesis was confirmed at 6 months after implantation of the polypropylene frame artificial trachea, ${ }^{8}$ in the present study we noted the formation of new blood vessels as early as 3 months after implantation of the nitinol frame artificial trachea.

Because the artificial trachea was pre-embedded in the omentum, the revascularization process may have been stimulated soon after the second surgery. This rapid postimplantation revascularization may prevent ischemia at the 


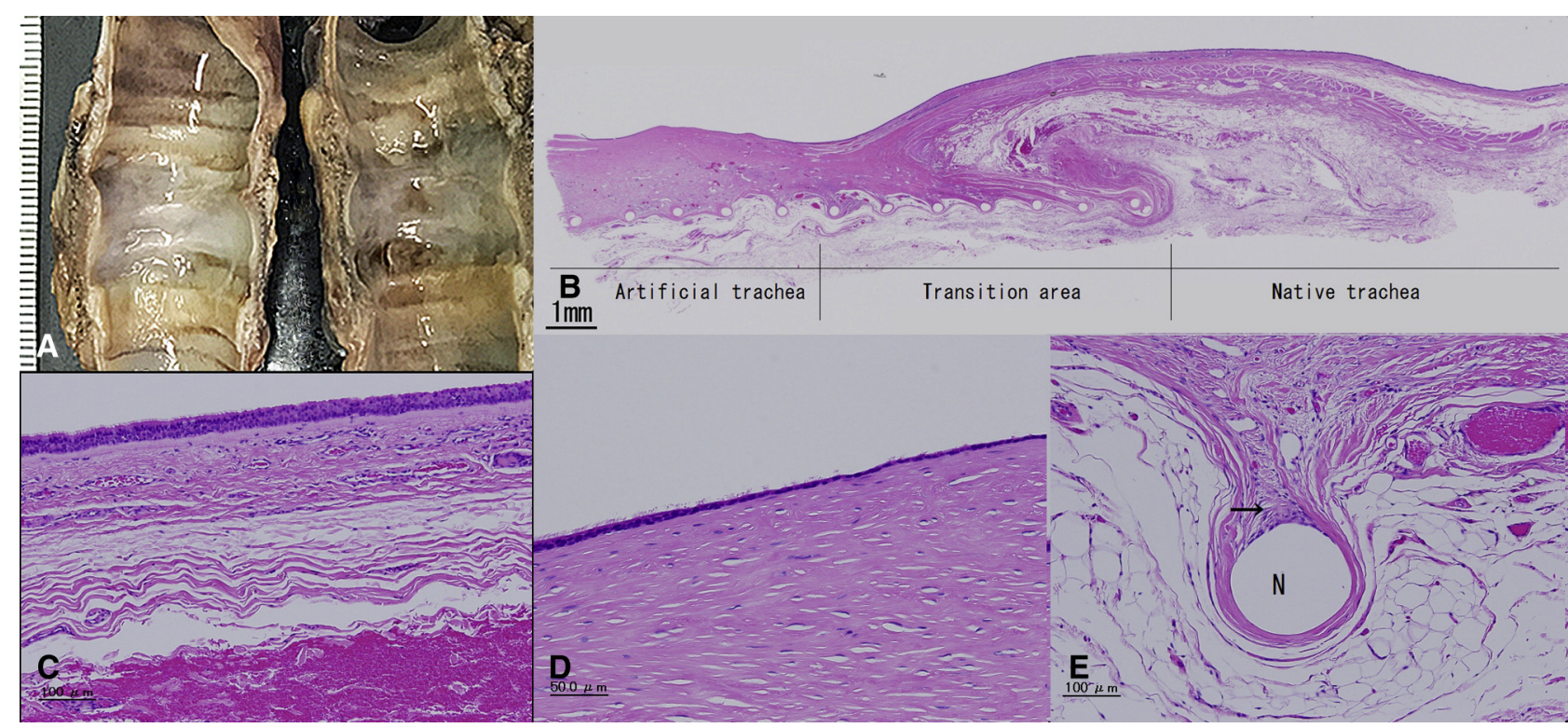

FIGURE 6. Macroscopic, microscopic, and histological findings of the resected artificial tracheae at 18 months after implantation. A, Macroscopic examination revealing no tracheal stenosis, with the artificial tracheal surface covered with normal mucosa. B, Microscopic examination showing the longitudinal transition from the native trachea to the artificial trachea. The smooth continuity of the tracheal epithelium to the artificial tracheal lumen was confirmed, but the muscular layer had not regenerated in the artificial trachea. C, A section of the native trachea revealing a stratified epithelial structure and secretory glands. D, A section of the artificial trachea revealing a monolayer airway epithelium with no secretory glands. E, Microscopic examination showing slight inflammation and scarring (arrow) around the nitinol frame $(\mathrm{N})$.

anastomosis site, thereby reducing the risk of stenosis. Although Liu and colleagues ${ }^{30}$ proposed that the omentum wraps could be omitted, their conclusion was based on shorter observation periods ( $<12$ months), and approximately one-half of their cases exhibited anastomotic stenosis. Therefore, we consider the use of omentum wrapping to be integral to our protocol. In this protocol, the placement period of the artificial trachea in the omentum was set at 3 weeks. In a preliminary experiment, the procedure was conducted with a 4-week placement period, but the omentum became strongly wrapped around the artificial trachea, and optimizing the use of the pedunculated blood vessels proved difficult. In addition, we were unable to obtain a sufficient length of lumen to perform telescopic anastomosis. In contrast, with a 2-week placement period, the artificial trachea was insufficiently embedded in the omentum and could be easily detached. In that state, the seal of the artificial trachea could not be ensured. Therefore, a short placement period would produce the same nonoptimal results as a single-stage surgery. These omentum-related problems did not occur with the 3 -week placement period.

In another preliminary experiment, we attempted to reconstruct the cervical trachea without omentum wrapping, but the collagen sloughed off from the artificial trachea soon after implantation, and the experiment failed. Nevertheless, it should be noted that the need for the second surgery and the 3-week waiting period before tracheal implantation are likely to place a heavy burden on patients. Coating the artificial endotracheal lumen with a bioabsorbable polymer ${ }^{8}$ or using stem cells with a bioreactor may eliminate the need for a second surgery. Alternatively, performing the implantation surgery and omental wrapping concomitantly may allow completion of the procedure in a single surgery.

In this study, we reconstructed a short segment mainly to confirm the biocompatibility of the artificial trachea. However, it is necessary to first resolve the aforementioned issues surrounding the second surgery and confirm the safety of long-segment reconstruction before this artificial trachea can be considered for clinical applications in humans. This study provides benchmarks for epithelialization and angiogenesis for future studies, and further evaluations should also consider the mucus clearance rate of the artificial trachea.

This study has the following limitations. First, there was a relatively small number of experimental subjects, and a larger sample will be needed in downstream analyses. Second, the current protocol requires a second surgery, which may present a heavy burden to patients. Third, the artificial trachea was relatively short, which currently limits the length of tracheal resection. Finally, the follow-up period was of insufficient duration when considering potential clinical applications to humans.

\section{CONCLUSIONS}

We have developed a novel artificial trachea composed of a nitinol frame that closely reproduces the physical characteristics of the native trachea. Using canine models, we have 
confirmed cell engraftment, good biocompatibility, and survival of 18 months or longer for this artificial trachea.

\section{Conflict of Interest Statement}

Authors have nothing to disclose with regard to commercial support.

We thank Ryo Marui for knitting the nitinol frame, Dr Akira Nakada for his advice regarding the collagen, Keiko Sakaguchi for drawing the schemata, and Satoshi Teramukai for his advice on the statistical analysis.

\section{References}

1. Kojima K, Vacanti C. Tissue engineering in the trachea. Anat Rec (Hoboken). 2014;297:44-50

2. Grillo HC. Tracheal replacement: a critical review. Ann Thorac Surg. 2002;73: 1995-2004.

3. Nakamura T, Sato T, Araki M, Ichihara S, Nakada A, Yoshitani M, et al. In situ tissue engineering for tracheal reconstruction using a luminar remodeling type of artificial trachea. J Thorac Cardiovasc Surg. 2009;138:811-9.

4. Sato T, Nakamura T. Tissue-engineered airway replacement. Lancet. 2008;372: 2003-4.

5. Omori K, Tada Y, Suzuki T, Nomoto Y, Matsuzuka T, Kobayashi K, et al. Clinical application of in situ tissue engineering using a scaffolding technique for reconstruction of the larynx and trachea. Ann Otol Rhinol Laryngol. 2008;117:673-8.

6. Teramachi M, Okumura N, Nakamura T, Yamamoto Y, Kiyotani T, Takimoto Y, et al. Intrathoracic tracheal reconstruction with a collagen-conjugated prosthesis: evaluation of the efficacy of omental wrapping. J Thorac Cardiovasc Surg. 1997; 113:701-11.

7. Teramachi M, Nakamura T, Yamamoto Y, Kiyotani T, Takimoto Y, Shimizu Y. Porous-type tracheal prosthesis sealed with collagen sponge. Ann Thorac Surg. 1997;64:965-9.

8. Sato T, Araki M, Nakajima N, Omori K, Nakamura T. Biodegradable polymer coating promotes the epithelization of tissue-engineered airway prostheses. $J$ Thorac Cardiovasc Surg. 2010;139:26-31.

9. Sekine T, Nakamura T, Matsumoto K, Liu Y, Ueda H, Tamura N, et al. Carinal reconstruction with a Y-shaped collagen-conjugated prosthesis. J Thorac Cardiovasc Surg. 2000;119:1162-8.

10. Nakada A, Fukuda S, Ichihara S, Sato T, Itoi S, Inada Y, et al. Regeneration of central nervous tissue using a collagen scaffold and adipose-derived stromal cells. Cells Tissues Organs. 2009;190:326-35.

11. Nakada A, Shigeno K, Sato T, Hatayama T, Wakatsuki M, Nakamura T. Optimal dehydrothermal processing conditions to improve biocompatibility and durability of a weakly denatured collagen scaffold. J Biomed Mater Res B Appl Biomater. 2017; 105:2301-7.

12. Delaere P, Van Raemdonck D. Tracheal replacement. J Thorac Dis. 2016;8(Suppl 2):S186-96.
13. Wood MW, Murphy SV, Feng X, Wright SC Jr. Tracheal reconstruction in a canine model. Otolaryngol Head Neck Surg. 2014;150:428-33.

14. Gonfiotti A, Jaus MO, Barale D, Baiguera S, Comin C, Lavorini F, et al. The first tissue-engineered airway transplantation: 5-year follow-up results. Lancet. 2014; 383:238-44.

15. Seguin A, Martinod E, Kambouchner M, Campo GO, Dhote P, Bruneval P, et al. Carinal replacement with an aortic allograft. Ann Thorac Surg. 2006;81: 1068-74.

16. Liu Y, Nakamura T, Shimizu Y, Ueda H, Yoshitani M, Toba T, et al. Tracheal allotransplantation in beagle dogs without immunosuppressants. Ann Thorac Surg. 2001;72:1190-4.

17. Agathos EA, Tomos P, Lachanas E, Gakiopoulou H, Pantopoulou A, Perrea D Experimental replacement of pig trachea with novel bioprosthesis from harp seal. Asian Cardiovasc Thorac Ann. 2010;18:557-62.

18. Neville WE, Bolanowski JP, Kotia GG. Clinical experience with the silicone tracheal prosthesis. J Thorac Cardiovasc Surg. 1990;99:604-12; discussion $612-3$.

19. Toomes H, Mickisch G, Vogt-Moykopf I. Experiences with prosthetic reconstruction of the trachea and bifurcation. Thorax. 1985;40:32-7.

20. Chang JW, Park SA, Park JK, Choi JW, Kim YS, Shin YS, et al. Tissue-engineered tracheal reconstruction using three-dimensionally printed artificial tracheal graft: preliminary report. Artif Organs. 2014;38: E95-105.

21. Kim J, Suh SW, Shin JY, Kim JH, Choi YS, Kim H. Replacement of a tracheal defect with a tissue-engineered prosthesis: early results from animal experiments. J Thorac Cardiovasc Surg. 2004;128:124-9.

22. Szycher M, Siciliano AA, Reed AM. Polyurethanes in medical devices. Med Des Mater. 1991;1:18-25.

23. Guidoin R, Couture J, Assayed F, Gosselin C. New frontiers of vascular grafting. Int surg. 1988;73:241-9.

24. Duerig TW, Pelton AR, Stöckel D. The utility of superelasticity in medicine. Biomed Mater Eng. 1996;6:255-66.

25. Shabalovskaya SA. On the nature of the biocompatibility and on medical applications of NiTi shape memory and superelastic alloys. Biomed Mater Eng. 1996; 6:267-89.

26. Stöckel D, Pelton A, Duerig T. Self-expanding nitinol stents: material and design considerations. Eur Radiol. 2004;14:292-301.

27. Barras CD, Myers KA. Nitinol - its use in vascular surgery and other applications. Eur J Vasc Endovasc Surg. 2000;19:564-9.

28. Sharpey-Schafer EP. Effects of coughing on intrathoracic pressure, arterial pressure and peripheral blood flow. J Physiol. 1953;122:351-7.

29. Gayrard P, Charpin J. Evaluation of the role of the large bronchi in the genesis of air obstruction in normal subjects and in various diseases. Am Rev Respir Dis. 1968;97:1076-88.

30. Liu Y, Lu T, Zhang Y, Qiao Y, Xi J, Wang Q. Collagen-conjugated tracheal prosthesis tested in dogs without omental wrapping and silicone stenting. Interact Cardiovasc Thorac Surg. 2016;23:710-5.

Key Words: artificial trachea, nitinol frame, physical characteristics, biocompatibility, tracheal reconstruction 